\title{
Acceptance and Commitment Therapy for Adults with ADHD during COVID-19: An Open Trial
}

\author{
Tim Fullen1*, Nazmeen Galab1, Karl A. Abbott' ${ }^{1}$, Marios Adamou ${ }^{2}$ \\ ${ }^{1}$ Adult ADHD and Autism Service, South West Yorkshire Partnership Foundation Trust, NHS, Manygates Clinic, \\ Wakefield, UK \\ ${ }^{2}$ School of Human and Health Sciences, University of Huddersfield, Huddersfield, UK \\ Email: ^tim.fullen@swyt.nhs.uk
}

How to cite this paper: Fullen, T., Galab, N., Abbott, K.A. and Adamou, M. (2020) Acceptance and Commitment Therapy for Adults with ADHD during COVID-19: An Open Trial. Open Journal of Psychiatry, 10, 205-215.

https://doi.org/10.4236/ojpsych.2020.104017

Received: September 10, 2020

Accepted: October 17, 2020

Published: October 20, 2020

Copyright $\odot 2020$ by author(s) and Scientific Research Publishing Inc. This work is licensed under the Creative Commons Attribution International License (CC BY 4.0).

http://creativecommons.org/licenses/by/4.0/

\begin{abstract}
This exploratory study reports the outcomes of a remotely delivered manualised form of Acceptance and Commitment Therapy (ACT) to adults with Attention Deficit Hyperactivity Disorder (ADHD) during the COVID-19 imposed "lockdown". Adults completing this intervention $(\mathrm{N}=12)$ reported statistically and clinically significant improvements on outcomes relating to self-reported $\left.\operatorname{mood} \mathrm{F}(1.356,14.917)=21.64, \mathrm{p}<0.001, \eta^{2}=0.66\right)$, anxiety $\left.\mathrm{F}(2,22)=22.93, \mathrm{p}<0.001, \eta^{2}=0.68\right)$, psychosocial adjustment $\left(\chi^{2}(4)=26.51\right.$, $\mathrm{p}<0.001)$ and treatment acceptability was good. Preliminary findings suggest this cost effective and remotely delivered psychotherapeutic approach might be one appropriate method for supporting the well-being and adjustment of adults with ADHD during future COVID-19 or other pandemic related lockdowns. Further research is urgently required to establish efficacy using larger sample sizes and the implementation of wait list and treatment comparison groups. Future research might also explore the use of this manualised approach in other clinical subgroups and the general population.
\end{abstract}

\section{Keywords}

ADHD, COVID-19, Coronavirus, ACT, Adjustment, Psychotherapy

\section{Introduction}

Attention Deficit Hyperactivity Disorder (ADHD) is one of the most common neurodevelopmental disorders with worldwide prevalence estimates in the adult population ranging widely from $0.1 \%$ up to $8.1 \%$ [1]. ADHD is characterised by deficits in attention and hyperactivity/impulsivity which persist across the deve- 
lopmental course and are pervasive [2]. In addition to primary neuro-behavioural symptoms, ADHD is a condition associated with a range of secondary psychosocial risk factors. Adults with this condition are more likely to be unemployed, to have a high turn-over of jobs and be employed in lower status occupation than their neuro-typical counterparts [3]. Qualitative research has frequently attested to the experiences of marginalisation in this cohort and adults with the condition are more likely than those from the general population to experience one or more psychiatric problems [4] [5]. Major depression is the single most commonly co-occurring psychiatric disorder presenting in 35\% - 50\% of the patient population. This is followed by one or more anxiety disorder occurring in $40 \%-60 \%$ of the patient population [5]. Adults with ADHD are therefore at an increased risk of developing psychiatric problems when compared to the general population.

The novel coronavirus termed COVID-19 was first reported in Wuhan (Hubei China) in December 2019. COVID-19 quickly spread throughout the globe resulting in the implementation of national "lockdowns" in which restrictions were placed on an individual's ability to travel, work and socialise in an effort to curb the spread of contagion. In the UK national lockdown was imposed on March $23^{\text {rd }}, 2020$. The implementation of this lockdown had immediate and profound consequences to individual's economic and social circumstances. National surveys alluded to the potential repercussions of this pandemic on the psychological well-being of the population with high levels of depression, anxiety and stress being reported [6]. It is anticipated these effects will be amplified in cohorts of vulnerable people such as those already experiencing, or at a heightened risk of experiencing, psychiatric problems.

The full extent of the psychological impact of COVID-19 on the general population and various sub-groups remains unclear although psychiatrists in the UK have warned of a potential "tsunami of mental illness" following the pandemic [7]. The cost and resource implications of addressing the mental health pandemic post COVID-19 defies calculation but the burden on services is expected to be significant [8]. The immediate and pending mental health crisis precipitated by the COVID-19 pandemic urgently requires the development of different psychotherapeutic approaches which can be delivered remotely, at scale and in a cost-effective fashion. These approaches should be tailored towards facilitating the adjustment and psychological resilience of individuals to a continued environment of uncertainty amidst the prospect of repeated lockdowns and restrictions on individual liberties.

Acceptance and Commitment Therapy (ACT) is one "third wave" psychotherapeutic approach which focusses on radically accepting personal experiences beyond the individual's control and taking committed action towards personal values [9]. This approach comprises six principles aiming to facilitate psychological flexibility: acceptance, present-moment awareness, cognitive defusion, self-as-context, mindfulness, and committed action. This approach is designed to be used flexibly based on individual needs and circumstances. ACT has been 
extensively researched and has demonstrated efficacy in reducing psychological distress and promoting acceptance in the context of various health and psychological conditions [10] [11]. The ACT approach may have promise in facilitating psychosocial adjustment in the context of COVID-19 because unlike other widely used time limited approaches, such as cognitive behavioural therapy [12], the onus is on adaptation and psychological flexibility rather than change.

The current study is one of the first to explore the potential viability of a costeffective programme of psychotherapeutic adjustment for individuals at risk of developing mental health problems in the context of the COVID-19 pandemic. This study reports the preliminary findings from a brief psychotherapeutic manual which was administered remotely via telephone and video conferencing. This brief three session manual is a novel addition to the field and treatment duration was based on the premise that important therapeutic processes including fostering patient insight and awareness, encouraging behavioural change, activity monitoring and relapse prevention could be facilitated [13], whilst still representing a cost-effective and accessible treatment option. This manual was informed by the ACT approach to facilitate the psychosocial adjustment of adults with ADHD to the COVID-19 pandemic and the restrictions implemented following lockdown. It is anticipated this manual might provide one effective, economically viable approach that can be delivered at scale by services seeking to prevent the deterioration of their patient's mental health in the event of future spikes of COVID-19 or other pandemics requiring lock down. The research question for this study was whether a remotely delivered ACT informed psychotherapeutic manual might be one feasible option for supporting the psychotherapeutic adjustment of adults with ADHD amidst the COVID-19 lockdown.

\section{Methods}

\subsection{Participants}

The convenience sample in this study was drawn from the South West Yorkshire Partnership Foundation Trust Adult ADHD and Autism Service case load. This service provides multi-disciplinary interventions to adults under the age of 25 with an established diagnosis of ADHD in adulthood. Initially 94 individuals were contacted on the telephone as part of routine checks during the COVID-19 lock down in order to complete a well-being survey. Each individual was contacted on three occasions during May 2020 and if they could not be reached it was deemed that they did not wish to complete the survey. Of the 94 participants contacted 24 completed a well-being survey and were also offered the opportunity to receive a psychotherapeutic intervention to help them adjust to the COVID-19 pandemic. This intervention was delivered between May and June 2020. From the 24 participants that completed the survey 13 opted into the intervention. A total of 12 participants completed the manual. This included 8 males and 4 females. The mean age for males was $22.63(S D=1.60)$ and 20.50 for females $(S D=1.91)$; with a total $(\mathrm{N}=12)$ mean age of $21.92(S D=1.93)$. 


\subsection{Experimental Design}

Patients who received intervention were part of an open trial involving three sessions delivered over the phone or via video conferencing. These sessions varied in length ranging from 45 - 90 minutes depending on the individual. Patients also received a follow up session to monitor progress and need for further input. A within participant design was employed with data collected at five time points including pre and at two weeks follow up.

\subsection{Measures}

The primary outcome measures administered were the Public Health Questionnaire (PHQ-9) and The Generalised Anxiety Disorder-7 (GAD-7). The PHQ-9 is a brief self-report measure designed to detect low mood and depression in primary care settings. It is widely used in clinical practice and has been extensively researched across clinical and non-clinical populations demonstrating good sensitivity (88\%) and specificity (88\%) [14]. The GAD-7 questionnaire is also a brief self-report questionnaire designed to measure anxiety severity in out-patient settings. Like the PHQ-9 it is widely used in clinical practice and has been extensively researched across clinical and non-clinical settings demonstrating good sensitivity (89\%) and acceptable specificity (82\%) [15].

The Brief Adjustment Scale-6 (BASE-6) is a novel measure of psychosocial functioning. The BASE- 6 comprises six questions rated out of 7 on the basis of severity. This rating scale was developed for session by session outcome measurement and has excellent internal consistency $(a=0.87-0.92)$ and reasonable test-retest reliability (0.76) based on a comprehensive sample including clinical and non-clinical populations [16].

\subsection{Psychotherapeutic Manual Administration Procedure}

The manual was based on an ACT protocol but included elements of psychoeducation and exercises drawn from compassion focussed (CFT) and cognitive behavioural therapy (CBT). In terms of CBT content Session 2 included a depiction of the thought, feeling and behaviour cycle to promote an awareness of the interdependence of external and internal factors maintaining distress. This session also included the "Pink Elephant" cognitive avoidance exercise often used within CBT treatment programmes. In regards CFT, Session 3 draws upon the skills training element of this approach to encourage participants to develop a positive self-script, adopt the position of a compassionate observer, and to be mindful.

Exercises and content were selected on the basis they promoted the process of adjustment and were suitable to be delivered remotely. The patient manual was posted the week before sessions began. The therapist's manual was identical to that of the patients but included questionnaires, prompts and a script to be followed. Patients and therapists both referred to their respective manuals during sessions. 
The first session includes content relating to self-care for adults with ADHD in the context of COVID-19 followed by a description of the ACT model using the "Three Circles" metaphor. A values questionnaire is then administered prompting a conversation to clarify the patient's core values. A simplified version of the "Hexaflex" known as the "Triflex" is then presented [17]. Home-work tasks are provided which include reflecting on values and identifying barriers to living a life consistent with these.

The second session includes a review of personal values followed by the introduction of a choice point and discussion of barriers identified during the week. The concept of mindfulness is then presented together with guided practice. The "Pink Elephant" cognitive avoidance exercise is presented as a means of demonstrating the futility of struggling from thoughts and negative experiences. Strategies for developing a compassionate stance to self are reviewed and mindfulness exercises are set for homework. Links to online mindfulness resources are provided.

The final session concerns opening up to experience and the "Quicksand" metaphor is explored followed by a mindfulness exercise. The concept of "Cognitive Defusion" or "Unhooking" is then presented with a discussion of various strategies for enabling this process. Key points and aspects of the manual are then reviewed and summarised.

\subsection{Therapists}

The team of therapists comprised three Assistant Psychologists, one Primary Well-being Practitioner and one Occupational Therapist. To ensure treatment fidelity all therapists received training and weekly supervision from the lead researcher and programme developer who is a Consultant Clinical Psychologist trained to Doctorate level.

\subsection{Analytic Approach}

This preliminary study sought to understand the potential benefits of a therapeutic manual to patients over time. A repeated measure ANOVA was conducted to observe change on the primary outcomes at pre, post and follow up intervals.

\section{Results}

\subsection{Treatment versus Non-Treatment Group Characteristics}

A between-groups one-way ANOVA was conducted to compare pre-intervention PHQ-9, GAD-7 and BASE-6 scores between those who opted to complete the ACT manual and those who did not (Table 1). All scores were normally distributed (Shapiro-Wilk, $\mathrm{p}>0.05$ ). Results of the ANOVA did not reveal any significant difference between groups at pre-intervention on the GAD-7 $(\mathrm{F}(1,22)=$ 3.06, $\left.\mathrm{p}=0.092 ; \eta^{2}=0.12\right)$, PHQ-9 $\left(\mathrm{F}(1,22)=3.43, \mathrm{p}=0.077, \eta^{2}=0.14\right)$ or BASE-6 $\left(\mathrm{F}(1,22)=0.49, \mathrm{p}=0.494, \eta^{2}=0.02\right)$. 
Table 1. Mean and standard deviation scores acquired pre-intervention, post-intervention $\&$ follow-up. ${ }^{\star} 1$ participant completed session one of intervention offered, but declined to continue further. This was added to did not complete intervention.

\begin{tabular}{ccccc}
\hline \multicolumn{5}{c}{ Pre-Intervention Scores } \\
\hline Pre-Intervention Survey $(N=24)$ & & GAD-7 & PHQ-9 & BASE-6 \\
\hline \multirow{2}{*}{ Did Not Complete Intervention $(N=12)$} & Mean & 9.25 & 8.42 & 15.75 \\
& $\sigma_{X}$ & 7.22 & 7.22 & 9.77 \\
Completed Intervention $(N=12)$ & Mean & 13.25 & 13.33 & 18.50 \\
& $\sigma_{X}$ & 5.05 & 5.69 & 9.58 \\
\hline
\end{tabular}

\subsection{Primary Outcome Measures}

PHQ-9 and GAD-7 scores of participants $(\mathrm{N}=12)$ who completed the ACT manual were collected at three interval points including pre-intervention (prior to delivery of the ACT Manual), post-intervention (after delivery of the last intervention session) and at two weeks follow-up (Table 2). All conditions adhered to a normal distribution (Shapiro-Wilk, $\mathrm{p}>0.05$ ).

Initial trend observations revealed a decline in PHQ-9 and GAD-7 mean scores from; pre-intervention (PHQ-9: $\mathrm{M}=13.33, \mathrm{SD}=5.69$; $\mathrm{GAD}-7$ : $\mathrm{M}=13.25$ $\mathrm{SD}=5.05$ ) to follow up (PHQ-9: $\mathrm{M}=7.33 \mathrm{SD}=5.09$; GAD-7: $\mathrm{M}=7.92, \mathrm{SD}=$ 5.37) after completion of the manual, with a total mean difference of 5.33 for GAD-7 and 6.00 for PHQ-9.

A within-subject ANOVA compared scores on the PHQ-9 and GAD-7 at three intervals (pre-intervention, post-intervention and follow-up). As Mauchly's Test indicated violations of sphericity, the Greenhouse-Geisser corrected statistic was used for PHQ-9 $\left(\chi^{2}(2)=6.44, \mathrm{p}=0.04\right)$ but not GAD-7 $\left(\chi^{2}(2)=\right.$ $0.99, \mathrm{p}=0.961)$. Results revealed a statistically significant effect of intervention on PHQ-9: Greenhouse-Geisser, $\left.\mathrm{F}(1.356,14.917)=21.64, \mathrm{p}<0.001, \eta^{2}=0.66\right)$ and GAD-7: Sphericity Assumed, $\left(\mathrm{F}(2,22)=22.93, \mathrm{p}<0.001, \eta^{2}=0.68\right)$. Bonferroni corrected comparison of individual conditions for GAD-7 revealed a significant decrease in scores (Figure 1) between pre-intervention and post-intervention $(\mathrm{p}=0.001)$ and pre-intervention and follow-up conditions $(\mathrm{p}<0.001)$. Similarly, a significant decrease in PHQ-9 scores was observed between pre-intervention and post-intervention conditions $(\mathrm{p}=0.009)$, pre-intervention and follow-up conditions $(\mathrm{p}<0.001)$ and additional post-intervention and follow-up conditions $(\mathrm{p}=0.048)$.

\subsection{Routine Outcome Measure}

Data generated from BASE-6 scores (Table 3) did not adhere to normal distribution within conditions of post intervention (Skew $=1.60$, Kurtosis $=1.45, \mathrm{~W}=$ $0.73, \mathrm{p}=0.001)$ and follow-up (Skew $=1.58$, Kurtosis $=1.39, \mathrm{~W}=0.72, \mathrm{p}=$ 0.001 ), therefore non-parametric analysis was employed and the median used as an alternative measure of central tendency. 


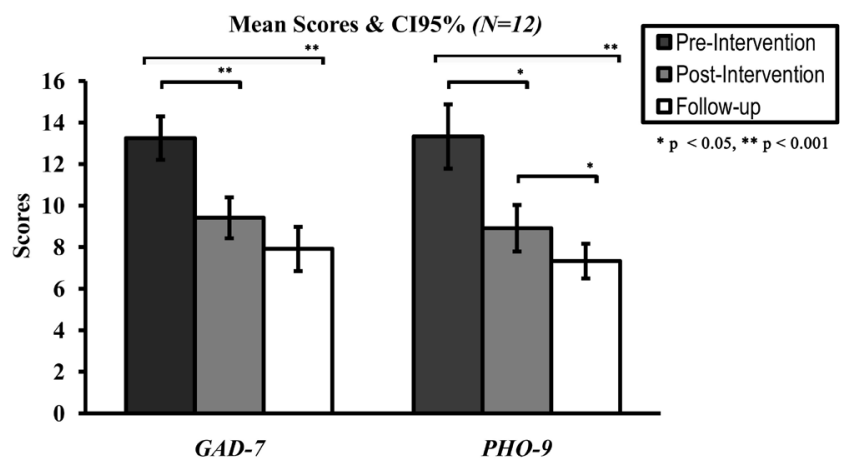

Figure 1. Visualisation of mean scores, $95 \%$ confidence interval error bars and significant differences (Bonferroni corrected) at ${ }^{\star} \mathrm{p}<0.05$ and ${ }^{\star *} \mathrm{p}<0.001$.

Table 2. Mean and standard deviation scores acquired pre-intervention, post-intervention \& follow-up.

\begin{tabular}{ccccc}
\hline \multicolumn{5}{c}{ Mean Intervention Scores $(N=12)$} \\
\hline \multirow{2}{*}{ GAD-7 } & Mean & Pre-Intervention & Post-Intervention (Session Three) & Follow-up \\
& $\sigma_{X}$ & 5.05 & 9.42 & 7.92 \\
& Mean & 13.25 & 6.13 & 5.37 \\
PHQ-9 & $\sigma_{X}$ & 5.69 & 8.92 & 7.33 \\
& & & 5.96 & 5.09 \\
\hline
\end{tabular}

Table 3. Median scores, quartiles and inter-quartile range across conditions.

\begin{tabular}{ccccc}
\hline \multicolumn{5}{c}{ Brief Adjustment Scale $(N=$ 12) } \\
\hline & 1st Quartile & Median & 2nd Quartile & IQR \\
\hline Pre-Intervention & 12.75 & $\mathbf{1 4 . 5 0}$ & 26.25 & $\mathbf{1 3 . 5 0}$ \\
Session One & 13.75 & $\mathbf{1 6 . 5 0}$ & 21.00 & $\mathbf{7 . 2 5}$ \\
Session Two & 9.75 & $\mathbf{1 2 . 0 0}$ & 18.75 & $\mathbf{9 . 0 0}$ \\
Post-Intervention (Session Three) & 7.50 & $\mathbf{8 . 0 0}$ & 13.50 & $\mathbf{6 . 0 0}$ \\
Follow-up & 6.00 & $\mathbf{7 . 0 0}$ & 12.25 & $\mathbf{6 . 2 5}$ \\
\hline
\end{tabular}

A general trend of reduction in scores was noted over the course of the ACT manual delivery from initial pre-intervention (Med. $=14.50, \mathrm{IQR}=13.50)$ to follow-up (Med. $=7, \mathrm{IQR}=6.25$ ), with a total difference of 6.75 ; despite initial first session increase of 2.00. Friedman's ANOVA was conducted to observe BASE- 6 scores across the 5 intervals (pre-intervention, session one, session two, post-intervention (session three) and follow up) with results displaying a significant main effect $\left(\chi^{2}(4)=26.51, \mathrm{p}<0.001\right)$.

Bonferroni corrected post hoc comparisons revealed significant decreases of BASE- 6 scores between pre-intervention and the follow-up condition $(\mathrm{p}<0.001$, $r=-0.60)$, and between session one and the follow-up condition $(p=0.001, r=$ -0.58). No other statistically significant pairwise comparisons were found following adjusted significance $(\mathrm{p}>0.05)$. 


\section{Discussion}

The aim of this preliminary study was to ascertain whether a brief, manualised and remotely delivered form of ACT might be a potentially effective means of facilitating the adjustment of adults with ADHD in the context of a pandemic and potential future lockdowns. The primary outcomes in this study pertained to adult's self-rated level of mood and anxiety. On the GAD-7 receipt of the ACT manual was associated with a statistically significant reduction in participants' self-reported anxiety levels between pre and post intervention which was maintained at follow up. Similarly, the PHQ-9 indicated a statistically significant decrease in self-reported levels of low-mood/depression in those receiving intervention. As with the GAD-7 gains were maintained at follow up. Those participants who provided improved scores on the PHQ-9 and GAD-7 typically selfrated as having improved from "moderately severe" to "moderate" range, or from "moderate" to "mild" range indicating change was also clinically significant.

The effect sizes for participants receiving the ACT manual were 0.68 for the GAD-7 and 0.66 for the PHQ-9 representing a sizeable change on these measures over the course of the intervention. A secondary outcome-based measurement system was administered each session to track adult's self-perception of their level of psychosocial adjustment (BASE-6). Overall, the BASE-6 demonstrated a statistically significant reduction in scores (increased psychosocial adjustment) in those receiving intervention overtime. A significant reduction in scores was observed between pre-intervention and the follow-up condition and between sessions one and the follow-up condition, both with large effect sizes. The rate of attrition for those opting into the intervention was also low $(<8 \%)$ indicating a high level of treatment acceptability and tolerance.

The ACT approach has been extensively researched and has demonstrated efficacy in reducing distress, promoting acceptance and increasing psychological flexibility and overall functioning in various patient groups. To date ACT has been shown to be efficacious in promoting adjustment and resilience in the context of various long-term health conditions such as epilepsy [18], multiple sclerosis [19], and chronic pain [20]. It has also proven an effective means of alleviating distress and improving functioning in patients with psychiatric disorders including anxiety and depression [21] and psychosis [22]. The benefits of this approach for facilitating adjustment to major life events such as bereavement have also been demonstrated [23]. Nevertheless, the current study is one of the first to explore the benefits and application of an ACT approach for facilitating adjustment in the context of pandemics and associated lockdowns.

The current study also extends recent research exploring the delivery of ACT using phone and web-based conferencing technology. ACT protocols have been administered in this form for various groups including people with Parkinson's [24], public speaking anxiety [25], and those engaged in smoking cessation work [26]. The results of the current study further support the suitability of the ACT approach to be delivered in a remote and manualised form. 
The results of this preliminary study also provide novel avenues to explore for the treatment of psychological distress in adults with ADHD more generally. To date research has demonstrated the value of other time limited approaches including Cognitive Behavioural Therapy, Dialectical Behaviour Therapy and Mindfulness in treating the primary and secondary symptoms of adult ADHD [27]. Nevertheless, the current study suggests that the ACT approach might prove to be a valuable addition to the field of adult ADHD more generally.

\subsection{Limitations}

Whilst this study is timely further research is required with greater methodological rigour. Importantly, future studies should include larger sample sizes to generate the necessary power and to ensure preliminary findings are not the result of sampling error. The inclusion of comparison treatment conditions would also allow for any therapist effects to be mitigated and the inclusion of treatment as usual control groups would also allow for further interpretation of the clinical significance of the intervention. Further research should therefore include a larger sample size, waitlist control and different treatment conditions. Finally, although this study was based on an ACT protocol it included a multi-model component drawing upon techniques from allied approaches including CFT and CBT. The inclusion of these components might be considered to undermine treatment integrity although the authors propose that, because these elements were used within a broader agenda of promoting psychological flexibility, their inclusion remains consistent with an ACT approach.

\subsection{Conclusion}

The current study is a preliminary enquiry exploring a cost-effective intervention that can be delivered remotely and at scale in the event of future spikes of COVID-19 or other pandemics. Preliminary results suggest this therapeutic programme is well tolerated and might prove effective in reducing anxiety, improving mood and facilitating psychosocial adjustment in adults with ADHD during future lockdowns. Further research is required to corroborate these preliminary findings. This research should include larger samples sizes and the use of waitlist and experimental control conditions to establish efficacy. Future research might also seek to corroborate these preliminary findings in different clinical sub-groups and with the general population.

\section{Conflicts of Interest}

The authors declare no conflicts of interest regarding the publication of this paper.

\section{References}

[1] Fayyad, J., Sampson, N.A., Hwang, I., et al. (2017) The Descriptive Epidemiology of DSM-IV Adult ADHD in the World Health Organization World Mental Health 
Surveys. Attention Deficit and Hyperactivity Disorders, 9, 47-65.

https://doi.org/10.1007/s12402-016-0208-3

[2] American Psychiatric Association (2013) Diagnostic and Statistical Manual of Mental Disorders (DSM-5 ${ }^{\circledast}$ ). American Psychiatric Pub. https://doi.org/10.1176/appi.books.9780890425596

[3] Holst, Y. and Thorell, L.B. (2019) Functional Impairments among Adults with ADHD: A Comparison with Adults with Other Psychiatric Disorders and Links to Executive Deficits. Applied Neuropsychology Adult, 27, 243-255. https://doi.org/10.1080/23279095.2018.1532429

[4] Schrevel, S.J., Dedding, C., van Aken, J.A. and Broerse, J.E. (2016) Do I Need to Become Someone Else? A Qualitative Exploratory Study into the Experiences and Needs of Adults with ADHD. Health Expectations, 19, 39-48. https://doi.org/10.1111/hex.12328

[5] Sobanski, E. (2006) Psychiatric Comorbidity in Adults with Attention-Deficit/ Hyperactivity Disorder (ADHD). European Archives of Psychiatry and Clinical Neuroscience, 256, i26-i31. https://doi.org/10.1007/s00406-006-1004-4

[6] Pierce, M., Hope, H., Ford, T., Hatch, S., Hotopf, M., John, A., Kontopantelis, A., Webb, R., Wessley, S., Mcmanus, M. and Abel, K.M. (2020) Mental Health before and during the COVID-19 Pandemic: A Longitudinal Probability Sample Survey of the UK Population. The Lancet Psychiatry. https://doi.org/10.2139/ssrn.3624264

[7] Royal College of Psychiatrists (2020) Press Release 15th May 2020. https://www.rcpsych.ac.uk/news-and-features/latest-news/detail/2020/05/15/psychi atrists-see-alarming-rise-in-patients-needing-urgent-and-emergency-care

[8] Thombs, B.D., Bonardi, O., Rice, D.B., Boruff, J.T., Azar, M., He, C., Marckham, S., Ying, S., Wu, Y. and Thombs-Vite, I. (2020) Curating Evidence on Mental Health during COVID-19: A Living Systematic Review. Journal of Psychosomatic Research, 133, Article ID: 110113. https://doi.org/10.1016/j.jpsychores.2020.110113

[9] Hayes, S.C. and Strosahl, K.D. (2004) A Practical Guide to Acceptance and Commitment Therapy. Springer Science \& Business Media, Berlin.

https://doi.org/10.1007/978-0-387-23369-7

[10] Lundgren, T., Dahl, J. and Hayes, S.C. (2008) Evaluation of Mediators of Change in the Treatment of Epilepsy with Acceptance and Commitment Therapy. Journal of Behavioral Medicine, 31, 225-235. https://doi.org/10.1007/s10865-008-9151-x

[11] Nordin, L. and Rorsman, I. (2012) Cognitive Behavioural Therapy in Multiple Sclerosis: A Randomized Controlled Pilot Study of Acceptance and Commitment Therapy. Journal of Rehabilitation Medicine, 44, 87-90.

https://doi.org/10.2340/16501977-0898

[12] Springer, K.S., Levy, H.C. and Tolin, D.F. (2018) Remission in CBT for Adult Anxiety Disorders: A Meta-Analysis. Clinical Psychology Review, 61, 1-8. https://doi.org/10.1016/j.cpr.2018.03.002

[13] Taylor, A., Tallon, D., Kessler, D., Peters, T.J., Shafran, R., Williams, C. and Wiles, N. (2020) An Expert Consensus on the Most Effective Components of Cognitive Behavioural Therapy for Adults with Depression: A Modified Delphi Study. Cognitive Behaviour Therapy, 49, 242-255. https://doi.org/10.1080/16506073.2019.1641146

[14] Kroenke, K., Spitzer, R.L. and Williams, J.B. (2001) The PHQ-9: Validity of a Brief Depression Severity Measure. Journal of General Internal Medicine, 16, 606-613. https://doi.org/10.1046/j.1525-1497.2001.016009606.x

[15] Spitzer, R.L., Kroenke, K., Williams, J.B. and Löwe, B. (2006) A Brief Measure for 
Assessing Generalized Anxiety Disorder: The GAD-7. Archives of Internal Medicine, 166, 1092-1097. https://doi.org/10.1001/archinte.166.10.1092

[16] Cruz, R.A., Peterson, A.P., Fagan, C., Black, W. and Cooper, L. (2019) Evaluation of the Brief Adjustment Scale-6 (BASE-6): A Measure of General Psychological Adjustment for Measurement-Based Care. Psychological Services, 17, 332-342. https://doi.org/10.1037/ser0000366

[17] Harris, R. (2013) Getting Unstuck in ACT: A Clinician's Guide to Overcoming Common Obstacles in Acceptance and Commitment Therapy. New Harbinger Publications, Oakland.

[18] Lundgren, T., Dahl, J., Yardi, N. and Melin, L. (2008) Acceptance and Commitment Therapy and Yoga for Drug-Refractory Epilepsy: A Randomized Controlled Trial. Epilepsy \& Behavior, 13, 102-108. https://doi.org/10.1016/j.yebeh.2008.02.009

[19] Pak, R. and Abdi, R. (2017) Effectiveness of Acceptance and Commitment Therapy (ACT) on Disease Acceptance and Experiential Avoidance in Patients with Multiple Sclerosis (MS). Contemporary Psychology, Biannual Journal of the Iranian Psychological Association, 12, 63-72.

[20] Wetherell, J.L., Afari, N., Rutledge, T., Sorrell, J.T., Stoddard, J.A., Petkus, A.J., Solomon, B., Lehman, D., Liu, L., Lang, A. and Atkinson, J.H. (2011) A Randomized, Controlled Trial of Acceptance and Commitment Therapy and Cognitive-Behavioral Therapy for Chronic Pain. Pain, 152, 2098-2107.

https://doi.org/10.1016/j.pain.2011.05.016

[21] Forman, E.M., Herbert, J.D., Moitra, E., Yeomans, P.D. and Geller, P.A. (2007) A Randomized Controlled Effectiveness Trial of Acceptance and Commitment Therapy and Cognitive Therapy for Anxiety and Depression. Behavior Modification, 31, 772-799. https://doi.org/10.1177/0145445507302202

[22] Bach, P. and Hayes, S.C. (2002) The Use of Acceptance and Commitment Therapy to Prevent the Rehospitalization of Psychotic Patients: A Randomized Controlled Trial. Journal of Consulting and Clinical Psychology, 70, 1129. https://doi.org/10.1037/0022-006X.70.5.1129

[23] Malmir, T., Jafari, H., Ramezanalzadeh, Z. and Heydari, J. (2017) Determining the Effectiveness of Acceptance and Commitment Therapy (ACT) on Life Expectancy and Anxiety among Bereaved Patients. Materia Socio-Medica, 29, 242. https://doi.org/10.5455/msm.2017.29.242-246

[24] Swalwell, C., Pachana, N.A. and Dissanayaka, N.N. (2018) Remote Delivery of Psychological Interventions for Parkinson's Disease. International Psychogeriatrics, 30, 1783. https://doi.org/10.1017/S1041610218000340

[25] Yuen, E.K., Goetter, E.M., Stasio, M.J., Ash, P., Mansour, B., McNally, E., Sanchez, M., Hobara, E., Fortea, S., Zulaicaa, K. and Watkins, J. (2019) A Pilot of Acceptance and Commitment Therapy for Public Speaking Anxiety Delivered with Group Video Conferencing and Virtual Reality Exposure. Journal of Contextual Behavioral Science, 12, 47-54. https://doi.org/10.1016/j.jcbs.2019.01.006

[26] Bricker, J.B., Bush, T., Zbikowski, S.M., Mercer, L.D. and Heffner, J.L. (2014) Randomized Trial of Telephone-Delivered Acceptance and Commitment Therapy versus Cognitive Behavioral Therapy for Smoking Cessation: A Pilot Study. Nicotine \& Tobacco Research, 16, 1446-1454. https://doi.org/10.1093/ntr/ntu102

[27] Fullen, T., Jones, S.L., Emerson, L.M. and Adamou, M. (2020) Psychological Treatments in Adult ADHD: A Systematic Review. Journal of Psychopathology and Behavioral Assessment, 42, 500-518. https://doi.org/10.1007/s10862-020-09794-8 